\title{
Entwicklung eines Prozesses zur Konstruktion von Hybrid-Im- plantaten für die Herstellung mittels additiver Fertigung
} \author{
Stelzer ${ }^{1}$ \\ ${ }^{1}$ Lehrstuhl für Konstruktionstechnik/CAD, Technische Universität Dresden \\ ${ }^{*}$ Korrespondierender Autor: \\ Martin Pendzik \\ Professur für Konstruktionstechnik/CAD \\ Institut für Maschinenelemente und Maschinenkonstruktion \\ George-Bähr-Staße 3c, 01062 Dresden \\ Telefon: 0351/463 34047 \\ Mail: martin.pendzik@tu-dresden.de
}

Martin Pendzik ${ }^{1 *}$, Lydia Mika ${ }^{1}$, Björn Scheibner ${ }^{1}$, Stefan Holtzhausen ${ }^{1}$, Ralph

\begin{abstract}
Additive manufacturing is characterized by a high degree of individuality and flexibility with regard to design and product layout. This enables the integration of different functions in a component. Application specific parameters such as load-bearing capacity, biocompatibility and manufacturing restrictions play a role of varying relevance. The combination of these parameters suitable for the individual patient case (implants) often cannot be realized with only one material. At time, however, only limited research has been done on design, manufacturing and application of hybrid implants (use of several materials). The aim is to develop and evaluate a process for designing hybrid implants depending on individual requirements and to clarify whether automation of this is possible.
\end{abstract}

\section{Keywords}

Hybrid-Implants, Individual-Implants, Implant-Design, ProcessAutomatisation for Hybrid-Implants, Additive Manufacturing 


\section{Einleitung}

\subsection{Motivation}

Fortschritte in der Fertigungstechnik ebnen neue Wege bei der Herstellung von Bauteilen und Produkten, welche den aktuellen Stand der Technik kontinuierlich erweitern. Zu diesen Technologien zählt auch die additive Fertigung.

Im Vergleich zu konventionellen Verfahren zeichnet sich die additive Fertigung durch ein hohes Maß an Individualität und Flexibilität hinsichtlich Design und Produktauslegung aus. Eine derartige Gestaltungsfreiheit ermöglicht die Integration unterschiedlicher Funktionen sowohl innerhalb eines Bauteils als auch auf dessen Oberfläche. Diese Eigenschaften sind in der Medizintechnik bei dem Einsatz von Implantaten von entscheidender Bedeutung. Anwendungsspezifisch spielen Parameter wie Belastbarkeit, Biokompatibilität und Fertigungsrestriktionen eine unterschiedlich relevante Rolle. Die für den individuellen Patientenfall geeignete Kombination dieser Parameter ist mit einem Werkstoff allein oftmals nicht realisierbar. Daher kann die Verwendung mehrerer Werkstoffe als hybrider Verbund sinnvoll sein. Der Vorteil eines solchen Hybrid-Systems, welches aus mindestens zwei verschiedenen Materialien besteht, liegt darin, dass das Implantat sowohl den Anforderungen an die mechanische Belastbarkeit als auch der Synthese zwischen Körper und Implantat gerecht werden kann. [1][2][3]

\subsection{Problemstellung}

Abgesehen von geschlechtsspezifischen Unterschieden ist die Anatomie des Menschen im Grundsatz gleich. Beschaffenheit, Form und Größe einzelner Knochen unterscheiden sich jedoch bei jedem Menschen. Kaum ein Knochen existiert in seiner Art identisch ein weiteres Mal [4]. Schlussfolgernd sind ebenfalls alle Knochendefekte, unabhängig von ihrer Entstehungsart, in ihrer Gestalt einzigartig. Daher muss in Situationen, die den Einsatz eines Implantats erfordern, ein individuell auf die Defektstelle zugeschnittenes Implantat hergestellt werden. Einen Leitfaden oder sogar automatisierten Prozess hierfür gibt es derzeit jedoch nicht.

Ferner ist bisher die Auslegung, Fertigung und Anwendung hybrider Implantate wenig erforscht. Herstellung und Konstruktion individueller Hybrid-Implantate stehen Herausforderungen wie dem konstruktiven und materiellen Aufbau, der individuellen Anpassung und der Stabilität des Designs gegenüber. Neben der fehlenden geometrischen Anpassung herkömmlicher Implantate, was u.a. für schlechtes Einwachsverhalten verantwortlich ist, werden bisweilen ungünstige Material(-kombinationen) verwendet. Um die Vorteile eines Hybridsystems jedoch ausschöpfen zu können, ist eine geschickte konstruktive Gestaltung und eine für den Individualfall geeignete Materialkombination zwingend notwendig.

\subsection{Forschungsziel}

In dieser Arbeit soll ein Prozess zur Konstruktionsmethodik vorgestellt werden, welcher zur Modellierung von hybriden Implantaten, unabhängig von der Defektstelle, Anwendung finden kann. Des Weiteren wird ein zweiter Prozess entwickelt, der basierend auf einem parametrischen Basismodell die Automatisierung eines Implantats ermöglichen soll.

Anhand eines Schädeldachdefekts wird der Prozess für individuelle hybride Implantate im Allgemeinen entwickelt und evaluiert. Im Zuge dessen werden Randbedingungen gemäß den individuellen Anforderungen und Fertigungsrestriktionen formuliert, das Implantat entsprechend in seine hybriden Elemente unterteilt und anschließend konstruiert sowie gefertigt. Hierbei soll die vollständige Prozesskette, von der Planung, über die Datenaufbereitung bis hin zur Konstruktion betrachtet werden. Das additiv gefertigte Implantat soll abschließend zur Prozessvalidierung genutzt werden und später in der zentralen Forschungsfrage, inwieweit eine Automatisierung von hybriden Implantaten sinnvoll ist, dem automatisierten Implantatbeispiel des Wirbelsäulen-Cages gegenüberstehen. 
Am Beispiel eines solchen Wirbelsäulen-Cages im Halswirbelbereich soll ein CAD-Modell entwickelt und als Basismodell verwendet werden, welches entsprechend der patientenindividuellen Anforderungen und auf der Grundlage von Schichtbilddaten parametrisch anpassbar ist. Hierfür wird eine patientenspezifische, lastangepasste Tragstruktur entwickelt, welche den Lastanforderungen im Bereich des Halswirbels entsprechen. Wie auch im individuellen Implantat, soll zusätzlich eine biokompatible Füllstruktur in das Implantat integriert werden. Beide Anwendungsbeispiele sind in Abbildung 1.1 skizziert.

Es gilt zu klären, wie die zuvor benannten Aspekte, wie u.a. Synthese zwischen Implantat und Körper, Stabilität des Designs und individuelle Anforderungen, sinnvoll und zielführend abgedeckt werden können und wie dies im konstruktiven Prozess umzusetzen ist. Weiterhin ist zu untersuchen, inwieweit eine Automatiserung der Konstruktion hybrider Implantate anwendbar und sinnvoll ist und ob dies einen generellen Lösungsansatz für Implantate unabhängig vom Anwendungsfall darstellen kann. Ziel ist es somit, einen Prozess zur Konstruktion hybrider Implantate in Abhängigkeit individueller Anforderungen zu entwickeln und zu evaluieren. Dabei soll die Frage diskutiert werden, ob ein Prozess allein ausreicht, um der Diversität der Anwendungsbereiche von hybriden Implantaten gerecht zu werden. Vor dem Hintergrund dieser Forschungsfragen werden die zwei erwähnten Defekte herangezogen, gegenübergestellt sowie die potenzielle Automatisierung der Implantate evaluiert und diskutiert.

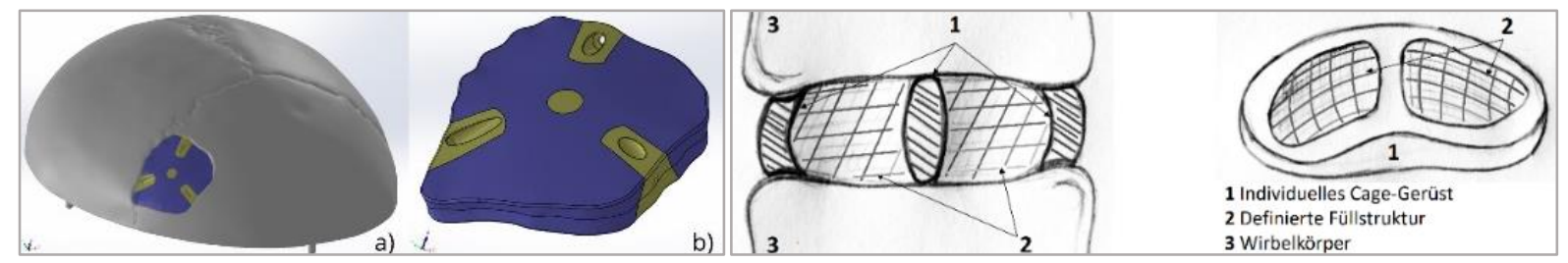

Abb. 1.1: Hybrides Schadelplatten-Implantat (I.) \& Skizzierung des hybriden Wirbelsäulen-Cages (r.)

\section{Stand der Technik}

\subsection{Individuelle Hybrid-Implantate}

Hybrid-Implantate kommen u.a. in der Mund-Kiefer-Gesichtschirurgie zum Einsatz und zeichnen sich durch vielfältige, positive Eigenschaften bzgl. Last- und Formanpassung aus. Dabei ermöglicht die erfolgreiche Entwicklung hybrider, individualisierbarer Implantate und der perspektivische Einsatz bei verschiedenen Behandlungen von geschädigten Knochen die bessere Integration in die körpereigene Knochenstruktur und vermeidet damit bisherige Defizite sowie Beschwerden des Patienten. Ferner kann der neuartige Versorgungsansatz die Anzahl notwendiger Folgeeingriffe, bspw. durch Revisionen, deutlich reduzieren und trägt damit zur Ressourcenschonung und zur Kostenreduktion im Gesundheitswesen bei. Nach [5] ergeben sich aus dieser hybriden Konstellation heraus allerdings noch ein paar konstruktiv zu beachtenden Kriterien, da bei der Kombination von zwei sehr unterschiedlichen Werkstoffen Versagensfälle auftreten können. Diese werden von den Hafteigenschaften der beiden Materialien und von im Bauteil vorliegenden Spannungen beeinflusst [5].

\subsubsection{Schädelplatten}

Mit Zunahme der fertigungs- und softwaretechnischen Möglichkeiten geht der Trend bei der Implantatherstellung mehr zu patientenindividuellen Implantaten. Lediglich in Einzelfällen werden herkömmliche Impantate wie Metallstege, -platten und Gitter verarbeitet. Diese stehen in diversen Variationen zur Verfügung und lassen sich leicht an die Defektstelle anpassen. Sie werden entsprechend der Defektgröße zugeschnitten und der Oberflächenkontur durch Verbiegen so nah wie möglich angeglichen. Die Vorteile bestehen in der sofortigen Verfügbarkeit 
und der einfachen Fixierung mittels Knochenschrauben. Die Methode ist jedoch nur eingeschränkt einsetzbar, da keine Rekonstruktion der Defektstelle im eigentlichen Sinn erfolgt.

Insbesondere für großflächige Knochendefekte, stellen Standardlösungen keinen adäquaten Ersatz dar. Derart größere oder, aufgrund ihrer besonderen Form, speziellere Defekte erfordern komplexere Implantate, welche exklusiv für eine Defektstelle modelliert und gefertigt werden. Ein Beispiel ist das Schädelimplantat „evo_SHAPE“ der Firma Evonos GmbH. Es handelt sich dabei um ein aus CT-Daten abgeleitetes, patientenspezifisches Implantat. Hervorzuheben sind hier das möglichst ästhetische Erscheinungsbild, die gute Passgenauigkeit und die mechanische Schutzfunktion. Als Werkstoff wurde Polyetheretherketon (PEEK) verwendet. Abbildung 2.1 zeigt die benannten Lösungen für Schädelimplantate. [5][6]

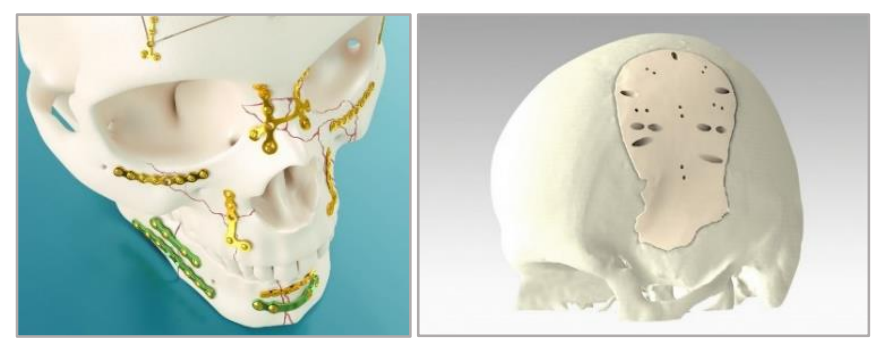

Abb. 2.1: Einsatzbeispiele für Schädelplattensysteme der Firma BIONIKA [7] (I.) \& Schädelimplantat evo_SHAPE der Firma Evonos GmbH [8] (r.)

\subsubsection{Wirbelsäulen-Cages}

Im Anwendungsbereich der operativen Behandlung von degenerativen Wirbelsäulen werden derzeit versteifende Maßnahmen getroffen (Spondylodese), wobei die Bandscheiben durch Implantate, sog. Cages, ersetzt werden. Aktuell bevorzugt werden in Serie hergestellte Cages in Standardgrößen (Abb. 2.2 a-c) [9], um die Abdeckung möglichst vieler Versorgungsfälle mit einer patientenorientierten Auswahl der Cages zu garantieren. Geometriebedingte Abweichungen können hierbei zu einem nicht optimalen Behandlungsergebnis für den Patienten führen. Ferner bieten Standard-Cages nur plane Anschlussflächen mit geringer Adaptionsmöglichkeit an die individuellen Wirbelflächen und -neigungen. Eine damit nicht sofortige, optimale Wirbelsegmentstabilisierung beeinträchtigt u.a. das Einwachsen des Knochengewebes [10]. Auch gleichmäßige, patientenspezifische Lastverteilung (Körpergewicht, -größe, Wirbelsäulenausrichtung, Aktivitätsgrad) zwischen stark beanspruchtem Wirbel und Cage wurden bisher nicht umgesetzt. Patientenspezifische, flächenindividuelle und lokal lastangepasste Cages aus Metallen (Ti64) wurden bereits vom Fraunhofer Institut für Lasertechnik (ILT) untersucht [11]. Der Fokus beim Design liegt auf der Kontur- und Flächenadaption an anliegende Wirbel sowie der offenporigen Gitterstrukturen im Implantatinneren (Abb. 2.2 d). Die damit an die Steifigkeit des anliegenden Knochens angepassten Cages bieten aufgrund ihrer patientenspezifischen Adaption ein hohes Potential beim Einwachsverhalten von Zellen sowie bei der Stabilisierung und können Standard-Cages im klinischen Alltag ersetzen [11]. Somit bestätigen sie die Vorteile der Individual-Cages, aber gleichzeitig auch Nachteile metallischer Implantate (vgl. K. 2.2.). Hybride Wirbelsäulen-Cages aus Metallen (z.B. Titan) und alternativen, knochenähnlichen Materialien bzw. Kunststoffen (z.B. PEEK) finden bisher kaum Anwendung. Somit werden entweder Knocheneigenschaften nicht optimal abgebildet oder das zu fördernde Knochenwachstum nicht optimal unterstützt. Die Folge sind Revisionsoperationen aufgrund zu geringer Stabilisierung des betroffenen Wirbelsäulenbereiches [12]. Hybride Implantatformen (Abb. 2.2 e) bieten jedoch das Potential durch die Kombination verschiedener Materialeigenschaften von Titan, PEEK und inertem Knochenzement (z.B. PMMA vgl. K. 2.2.) bisherige Defizite auszugleichen. Nach [12] wurde gezeigt, dass diese Cage-Gestaltung ein gutes Knochenwachstum, knochenähnliche Steifigkeiten sowie ein deutlich minimiertes Einsinken in den 
Knochen gewährleistet. Eine optimale konstruktive Verteilung von Titan und PEEK sowie die Möglichkeit zur kombinierten additiven Fertigung verschiedener Strukturen bleibt jedoch offen.
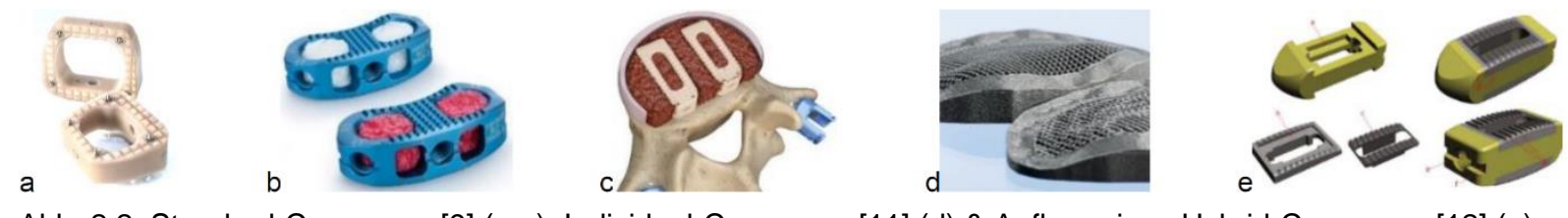

Abb. 2.2: Standard-Cages von [9] (a-c), Individual-Cages von [11] (d) \& Aufbau eines Hybrid-Cages von [12] (e)

\subsection{Material und Fertigung}

Der Einsatz von Implantaten begrenzte sich bisher auf die Verwendung kompakter metallischer, keramischer oder polymerer Rohlinge und die damit verbundenen Technologien zur Fertigung. Bei einigen Firmen sind Bestrebungen und Erfolge bei der Individualisierung von Implantaten aus Metall und Keramik erkennbar, die mittels additiver Fertigung hergestellt werden [13][14]. Als Vorteile gegenüber herkömmlichen Verfahren sind zu nennen: hohes Individualisierungspotential, zellulare Volumen- und Oberflächengestaltung während der Fertigung, ohne nachträgliche Beschichtung sowie Einsatz biokompatibler Werkstoffe, die nicht oder schwer zu spanen sind.

Gängige additive Fertigungsverfahren sind für metallische Werkstoffe (i.d.R. Ti64) das Laser- und Elektronenstrahlschmelzen (LBM, EBM). Diese Verfahren arbeiten durch das sequentielle Verschmelzen einzelner Pulverschichten in einem Pulverbett und sind daher in ihrer möglichen Designfreiheit nahezu unbegrenzt. Vor allem die Integration von rauen, strukturierten Oberflächen für das Verbessern des Einwachsverhaltens von Zellen ist als Vorteil zu nennen. Nachteilig ist, dass nach erfolgter Formgebung ein Sinterschritt und entsprechende Nachbearbeitungsstufen (z.B. Pulverresteentfernung) notwendig sind, was sich negativ auf Kostenstruktur, Qualität, Formenvielfalt, Oberflächenstruktur, Porosität und Biokompatibilität auswirkt. Die damit verarbeiteten inerten Materialien können nicht biologisch abgebaut und in körpereigenen Knochen umgewandelt werden. Ferner sind bei metallischen Implantaten Störungen des Kälteempfindens sowie der Verlust der Knochentragfähigkeit im Kontaktbereich aufgrund hoher Steifigkeitsunterschiede bekannt und verursachen somit hohe Punktlasten (stress-shielding). [1][15][16] Kunststoffe wie Polyetheretherketon (PEEK) finden seit einiger Zeit ebenfalls Verwendung in medizinischen Implantaten. PEEK ist inert, sterilisierbar und ähnelt in seinen mechanischen Eigenschaften dem humanen Knochen. Zusätzlich können Implantate aus Polymethylmethacrylat (PMMA) während der Operation angepasst werden. Nachteilig bei den Kunststoffen sind jedoch die Herstellungskosten und nicht vorhandene Integrationsmöglichkeiten des umliegenden Knochens, wodurch sie keine direkte Verbindung mit diesem eingehen. [17][18]

Implantate aus mehreren verschiedenen Komponenten werden ebenfalls angewendet oder erforscht. Im Bereich nichtindividueller Implantate gibt es Systeme, welche Hybrid-Implantate aus einer metallischen Grundstruktur mit einer biologisch kompatiblen Beschichtung (z.B. Calcium-Phosphat) anbieten. Auch existieren individualisierbare Implantate aus gitterartigen Titan-Tragstrukturen und biokompatibler Matrix [19]. Dagegen existieren pastöse Calciumphosphat-Präparationen (CPC), die mittels 3D-Druckverfahren die endkonturnahe Herstellung von zellularen Formkörpern ohne zusätzliche Nachbearbeitung erlauben [20][21][22][23]. Durch Einsatz von Füllstrukturen aus biokompatiblen Kunststoffen können komplexe endkonturnahe Implantate hergestellt werden [24]. Die positiven Eigenschaften bezogen auf das Integrationsverhalten von Zellen und der Absorbierbarkeit sind nachgewiesen [25]. Die Technologie ist zur Versorgung von nicht lasttragenden geschlossenen Knochendefekten geeignet [24][26]. Für den breiten Einsatz ist eine Kombination mit lasttragenden, individualisierten Implantatkomponenten aus Polymeren oder Titan erforderlich. Für die Kombination aus additiv gefertigten Trag- und Füllstrukturen (Knochenzement) gibt es bisher keine Lösungen. 


\subsection{Herstellungsprozess}

Der Herstellungsprozess patientenindividueller Implantate ist derzeit sehr zeit- und kostenaufwändig. So ist stets ein aufwendiger Diagnose- und Planungsprozess, ein erheblicher konstruktiver Aufwand und eine meist zeitintensive Fertigung notwendig. Eine Fokussierung auf eine konkrete Anwendung von individualisierbaren Implantaten ermöglicht eine deutliche Reduzierung des Aufwandes. Dass dies mit bspw. einem parametrischen Basis-Implantatmodell erfolgsversprechend umsetzbar ist, konnte bereits an verschiedenen Beispielen wie Kieferimplantaten [27], Knieendoprothesen [26] und Unterschenkelorthesen [28] bewiesen werden.

Ferner existieren derzeit weder ein definierter Konstruktionsprozess noch geläufige Richtlinien für die Konstruktion hybrider Implantate. Durch [5] konnten hierfür jedoch wesentliche Aspekte zusammengetragen werden: Für das hybride Implantatdesign selbst sind u.a. definierte Maße der Stützstruktur entsprechend der Last- und Knochenfälle, Füllstrukturen entsprechend der Knochendicke und -struktur sowie eine effiektive Kombination der beiden Implantatstrukturen zwingend zu beachten. Des Weiteren sind zusätzliche Schritte innerhalb der Prozesskette zu integrieren. Dazu gehören u.a. die Aufschlüsselung der Implantatkonstruktion in Teilschritte wie die Konstruktion von Auflagestrukturen, Stützstreben, Befestigungselementen und Draht- und Füllkomponenten sowie die separate aber gleichzeitig integrale Fertigung der beiden Implantatteilstrukturen (Trag- und Füllstruktur). [5]

\section{Verwendete Methoden und Vorgehensweisen}

\subsection{Generelles Vorgehen}

Der digitale Prozess beginnt mit der Ermittlung von patienten-spezifischen Daten (CT, MRT, etc.) und der Datenaufbereitung. Datenaufbereitung ist hierbei die Überführung der bspw. CTDaten in segmentierte sowie ableitbare 3D-Daten. Von diesen Daten ausgehend lassen sich Konturen, Oberflächen oder ganze 3D-Modelle in eine CAD-Software überführen und können für die Implantat-Konstruktion bereitgestellt werden. Mithilfe dieser Funktion wird der Randbereich der Defektstelle nachmodelliert, um davon das Implantatmodell abzuleiten. Diese Phase ist die Datenverarbeitung. Anschließend wird die Auslegung des Implantatmodells in der Planungsphase abhängig von dem Ort des Defektes und der patientenspezifischen Gegebenheiten definiert und in Trag- und Füllstruktur unterteilt. In der letzten Phase, der Modellierungsphase, werden aus dem Implantatmodell die hybriden Strukturen konstruiert. Bei der automatisierten parametrischen Implantatmodellierung findet durch den Parameterübertrag eine Anpassung des bereits bestehenden Basismodells statt, welches mittels dem zuvor erwähnten Prinzip konstruiert wurde. Die entwickelte Prozesskette ist der folgenden Abb. 3.1 zu entnehmen. Die Planungsphase ist lediglich für die vollständige Modellierung eines hybriden Implantats relevant, da bei der automatisierten Implantaterstellung das vorangehende Basismodell lediglich angepasst und verändert wird, jedoch keine Elemente neu konstruiert werden.

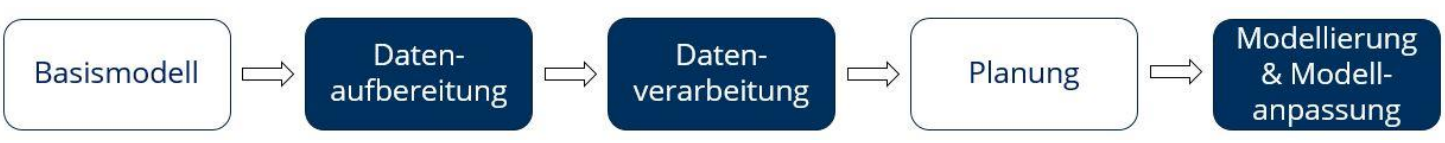

Abb. 3.1: Prozesskette zur Erstellung hybrider Implantate

\subsection{Konstruktionsprozess für ein individuelles hybrides Implantat}

\subsubsection{Methoden zur Überführung von Patientendaten (CT-Daten) zu Geometriemodel- len angestrebter Implantate}

Für die Erstellung eines Individualimplantats sind die Patientendaten in Geometriemodelle zu überführen. Üblicherweise wird zur Aufnahme der Patientendaten ein CT verwendet. Die 
daraus resultierenden CT-Daten, häufig in Form von DICOM- oder TIFF-Dateien, müssen in den Konstruktionsprozess eingebunden werden. Dafür wurde mit dem am Lehrstuhls Konstruktionstechnik/CAD der TU Dresden entwickelten Softwaretool CTinA gearbeitet, welches für die Konstruktion von hybriden Implantaten erweitert und evaluiert wurde. Anders als andere kommerzielle Tools, ermöglicht es den Zugriff auf die CT-Rohdaten direkt aus der CAD-Umgebung SolidWorks heraus. Neben dem Import und dem Anzeigen von Schichtbildern des Patienten, können definiert Oberflächenmodelle der Knochenstruktur importiert werden. Damit ist es dem Konstrukteur möglich direkt mit den CT-Daten zu konstruieren. Insbesondere bei der Konstruktion von Fixierungselementen hat der Konstrukteur Einblick in die Knochensituation des Patienten. Abbildung 3.2 zeigt die Kommunikation zwischen den Systemen.

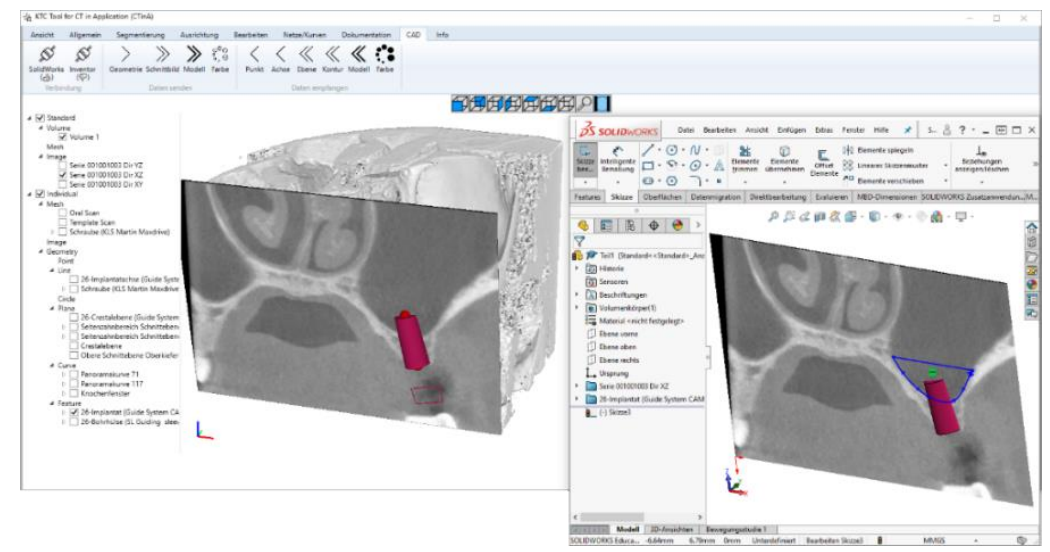

Abb. 3.2: Kommunikation zwischen dem Tool CTina und der CAD-Anwendung SolidWorks

\subsubsection{Entwicklung einer Methode zur Konstruktion des Implantatmodells und zur Auf- teilung dieses Modells in Trag- und Füllstruktur}

Um Geometriemodelle der angestrebten Implantate konstruieren zu können, wurde ein mehrstufiges Konstruktionsverfahren entwickelt, welches nachstehend aufgeführt ist:

1. Modellierung der Defektstelle

2. Rekonstruktion des Hüllvolumens des Implantates

3. Festlegung der Fixierungsstrategie

4. Konstruktion der geforderten Teilvolumina für Trag- und Füllstruktur

Im ersten Schritt wird die Defektstellengeometrie in CTinA abgeleitet. Hierfür werden ausreichend viele und in frei wählbarer Anzahl manuell Referenzpunkte auf der Knochenoberfläche in und um die Defektstelle als sogenannte 3D-Skizze erstellt (Abb. 3.3).
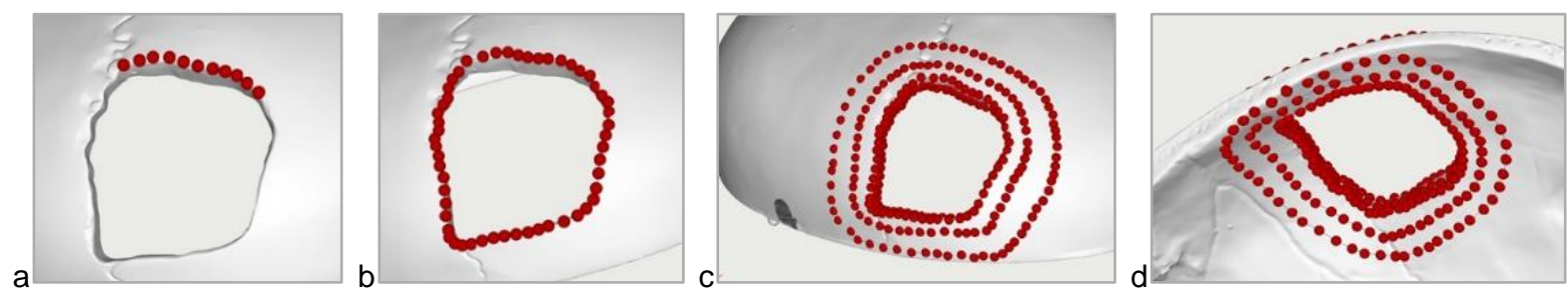

Abb. 3.3: Erzeugte Referenzpunkte auf Schädeloberfläche in CTinA (a); Geschlossene Polylinie (b); Geschlossene Polylinien auf Schädeloberfläche außen (c) \& Schädeloberfläche innen (d)

Diese Kurven sind so platziert, dass Kanten und Ränder der Defektstelle möglichst genau nachgebildet werden können. Die Ableitung der näheren Umgebung des Defekts ist ebenfalls 
von Relevanz, um für die im weiteren Verlauf erfolgende Integration von Befestigungselementen in das tragende Material ausreichend Kontaktfläche zu gewährleisten. Nach dem Erstellen der Referenzobjekte werden diese 3D-Kurven über die SolidWorks-Schnittstelle in CTinA an die CAD-Software übergeben. Über diese Kurven werden einzelne Oberflächen-Patches erzeugt und anschließend zu einer geschlossenen Oberfläche zusammengeführt. Der Volumenkörper der Defektstelle des Schädeldachdefektes ist in Abbildung 3.4 a dargestellt.

Im nächsten Schritt wird das Implantat-Volumen konstruiert. Dafür werden 3D-Skizzen mit Leitlinien eingefügt anhand derer die gekrümmte Implantatform rekonstruiert werden kann. Mithilfe dieser Leitlinien als Zwangsbedingung und der nachgebildeten Defektstelle als Flächenbegrenzung werden zwei Freiformflächen erstellt (jeweils eine auf der Innen- und der AuBenseite des Implantats). Die Abbildung 3.4 b-c zeigt die konstruierte innenliegende Fläche des Implantatvolumens sowie den finalen Volumenkörper, welcher die Basis für das hybriden Implantatgeometrien darstellt.
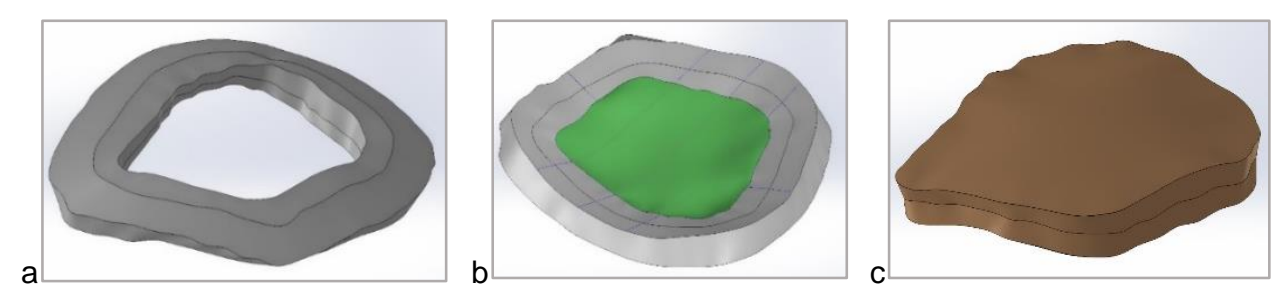

Abb. 3.4: Rekonstruierte Defektstelle als Volumenkörper (a); Fläche auf Implantatinnenseite (grün) \& Leitlinien (blau) (b); Volumenkörper des Implantats (c)

\subsubsection{Aufteilung des Implantats in seine hybriden Elemente}

Die Erzeugung der Hybridstruktur erfordert als vorhergehenden Schritt die Aufteilung der Hüllgeometrie in Trag- und Füllstruktur. Dabei muss als erstes die Form und Auslegung der Tragstruktur bestimmt werden. Hierfür sind folgende Randbedingungen erarbeitet worden:

- Baurichtung

- Beachtung geometrischer Besonderheiten (bspw. Schrägen an Kontaktflächen von Implantat und Knochen)

- Berücksichtigung der Implantations-Richtung

- Verwendung als Langzeit- oder Kurzzeitimplantat

- Defektkontur und -stelle (bspw. Schädel oder Jochbein)

○ Möglichkeiten zur Integration von Befestigungselementen (Anzahl, Ort, Eindringtiefe in den Knochen)

- Notwendiger Schutz gegen äußere mechanische Einwirkungen (Stoß, Schlag, Tragfähigkeit, etc.)

- Druckrichtung des CPC-Paste während der additiven Fertigung

○ Abhängig von Baurichtung und Beschaffenheit der Tragstruktur

- Bedingt Formschrägen der Tragstruktur

- Formschrägen

- Für Verbund von Trag- und Füllstruktur als Formschluss (dient als Auflagefläche wodurch Füllstruktur gegen Verrutschen gesichert ist)

- Fertigungsbedingung aufgrund der Extrusionsnadel, die bei dem Eindrucken der Füllstruktur verwendet wird

- Kontaktfläche von CPC-Füllstruktur zu Knochen

○ Zwingend so groß wie möglich, um Osseointegration zu gewährleisten

- Kontaktfläche Tragstruktur zu Füllstruktur

- Ausreichend groß wählen, um stabilen Verbund zu ermöglichen. 
Ausgehend von den aufgeführten Randbedingungen und der patientenspezifischen Anforderungen des Defektes wurde ein dreiarmiges Modell als Tragstruktur gewählt, um insbesondere sowohl der verhältnismäßig hohen Kontaktfläche von Knochen zu Füllstruktur, als auch der stabilen Verankerung im Schädel sowie dem Schutz gegen äußere Krafteinwirkungen gerecht zu werden. Ein Schwerpunkt bei hybriden Implantaten stellt die Integration von Befestigungselementen in das tragende Material dar. Grundsätzlich ist hierfür eine Schraubverbindung zu bevorzugen. Zur Konstruktion von Verschraubungen wurden Varianten erarbeitet, die es erlauben, Schrauben in der Tragstruktur zu versenken (Verwendung von Taschen), sodass diese nicht aus der Schädeldecke herausragen. Dies dient einerseits dem ästhetischen Zweck, vorrangig sollen damit jedoch Folgeverletzungen durch äußeren Kontakt vermieden werden. Zur Beschreibung der Geometrien für Tragstruktur und CPC-Paste wurden jeweils unterschiedliche Volumenmodelle verwendet. Diese ergeben sich durch boolesche Verschneidungen vom Hüllvolumen und der Geometrie der Tragstruktur. Beide Modelle lassen sich in einem Austauschformat exportieren und können durch additive Verfahren gefertigt werden. Die resultierende Konstruktion für das hybride Schädeldachimplantat ist in Abbildung 3.5 dargestellt.

a

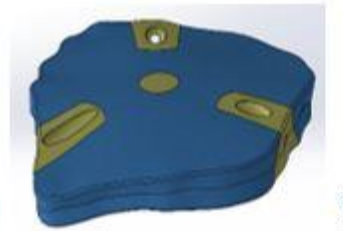

b

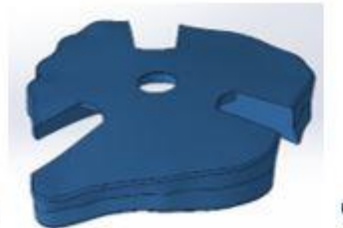

$\mathrm{C}$

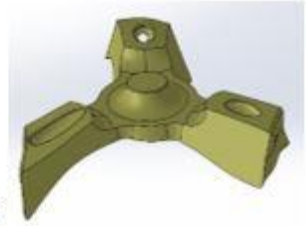

Abb. 3.5: Konstruierte Hybridstruktur des Implantats: vollständig (a); Tragstruktur (b); Füllstruktur (c.)

\subsection{Erstellungsprozess für ein parametrisch individuelles hybrides Implantat}

Neben der Methode aus Kapitel 3.2 wird eine parametrische individuelle Konstruktionsmöglichkeit von Implantaten vorgestellt. Grundlage für dieses Vorgehen bildet ein zuvor auf CTDaten konstruiertes Basismodell des Implantates (vgl. K. 3.1.) am Beispiel eines WirbelsäulenCages für den Bereich der Halswirbelsäule. Die inititale Konstruktion dieses Modells orientiert sich dabei am Aufbau bereits existierender Cages (vgl. K. 2.1.2). Als Unterschied und Optimierung zu diesen Cages werden der von Beginn an parametrische Aufbau und die entsprechende Konstruktionsweise gesehen, welche später die unkomplizierte, schnelle parametergesteuerte Anpassung des CAD-Basismodells erlauben sollen. Das dabei gezielte Umsetzen von steuerbaren (implantat- und defektstellen-spezifischen sowie veränderbaren) und nichtsteuerbaren (feststehenden, konstruktiven) Modell-Parametern erfolgt in einem iterativen Prozess zur passenden Integration der Patienten-Mensch-Parameter. Einbezogen werden dabei anatomische, geometrische und mechanische Parameter (Knochenmaße, Achstellungen, Belastungsgrad, etc.) sowie Metadaten (Aktivitätsgrad, Vorerkrankungen, etc.). Diese werden sowohl aus den in CTina abgeleiteten CT-Daten (vgl. K. 3.2.1) des Patienten als auch aus den vom Mediziner diagnostizierten Daten generiert.

Desweiteren ist die anschließende konstruktive, hybride Aufteilung des Implantates in zwei Komponenten (Trag- und Füllstruktur) hervorzuheben, welche sich an Kapitel 3.2. anlehnt. Beide Komponenten sind weiterhin parametrisch aufgebaut und beziehen dabei jeweils unterschiedliche, steuernde Parameter des Patienten sowie des Implantatmodells ein. Unterschieden wird hierbei in eine individuell- und vorangig lastangepasste Tragstruktur aus PEEK (bspw. mechanische und anatomische Parameter), welche den eigentlichen Cage als solches bildet sowie in eine individuell- und formangepasste Füllstruktur aus CPC (bspw. geometrische Parameter), welche die spezifischen geometrischen Formen und Wirbelneigungen nachempfindet. Somit wird ebenfalls sowohl die Trag- als auch die Füllstruktur für die kombinierte, additive Fertigung fest definiert. Angestrebt wird dabei ein integraler, reproduzierbarer Fertigungsprozess, welcher die Kombination von Füll- und Tragstruktur sicherstellt. 
Der Anpassungsprozess des Implantatmodells selbst wird in Anlehnung an [26][28][29] auf Wirbelsäulen-Cages als weiteren Anwendungsfalls übertragen, da sich der generelle, parameter-gesteuerte Prozess bereits bewährt hat. Kurz erläutert wurde dies bereits in Kapitel 3.1. und beschreibt im wesentlichen die Datenaufbereitung und -verarbeitung, die Fertigstellung des individuellen Implantatmodells durch patienten-spezifische parametrische Anpassung des Basismodells sowie dessen additive Fertigung. Diese Schritte können nach erfolgreich evaluierter, manueller Bedienung automatisiert werden und ermöglichen so einen zeit- und kostensparenden Herstellungsprozess für patienten-gerechte, individuelle (Hybrid-)Implantate. Diese finden bei Gewebeersatz Verwendung, welcher bei jedem Menschen ähnlich auftreten kann.

\section{Ergebnisse und Diskussion}

\subsection{Ergebnisse}

Auf Basis von CT-Daten wurden unterschiedliche Defektstellen abgeleitet und ein hybrides Implantat konstruiert. Konkret konnte am Beispiel eines Schädeldachdefektes ein Prozess zur Konstruktion eines hybriden Implantates, basierend auf einem individuellen und sich patientenübergreifend nicht wiederholenden Knochendefekt, aufgezeigt werden. Trag- und Füllstruktur wurden erfolgreich additiv gefertigt, wobei die Füllstruktur in die Tragstruktur eingedruckt wurde. Für eine formschlüssige Oberfläche beider Strukturen wurde eine Stützstruktur als Unterlage genutzt, die zusammen mit der Tragstruktur gefertigt wurde und das Negativ des Defekts bildet. Die Füllstruktur haftet an der Tragstruktur und löst sich bei kleinen äußeren Krafteinwirkungen nicht von dieser ab. Der Kontakt der Füllstruktur zum Knochen ist erheblich größer, als der der Tragstruktur zum Knochen. Auch die Verankerungsschrauben ragen nicht aus dem Implantat heraus. Das finale hybride Implantat erfüllt somit alle relevanten Anforderungen. Das Vorgehen kann daher als Grundlage für die Erstellung hybrider Implantate dienen. Die Abbildung 4.1 stellt die konstruierten und gefertigen hybriden Implantate gegenüber.

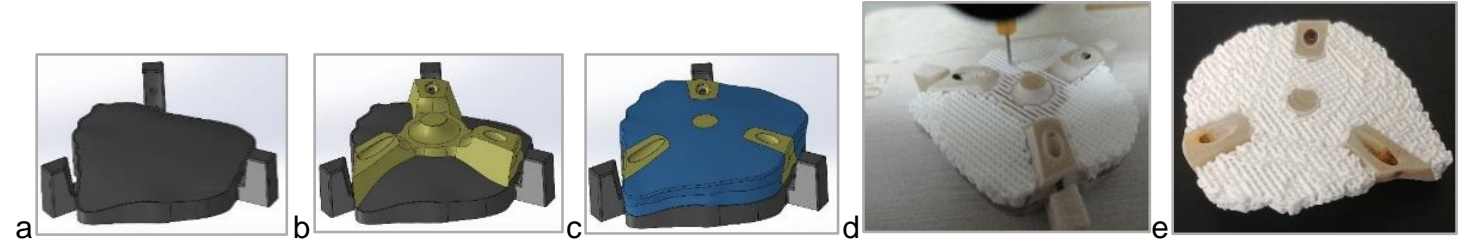

Abb. 4.1: Konstruiert: Stützstruktur (a), Stütz- und Tragstruktur (b) sowie Stütz-, Trag- und Füllstruktur (c); Eindruckprozess (d) und additiv gefertiges Hybrid-Implantat (e)

Es hat sich gezeigt, dass die Füllstruktur nach dem Aushärten auch stark an der Stützstruktur haftet und sich mitunter nur mit großem Aufwand von dieser trennen lässt. Dies kann auf die große Kontaktfläche beider Strukturen und die Eindruckstrategie zurückzuführen sein.

Des Weiteren wurde ein Prozess entwickelt, welcher ein Implantat, abhängig von den patientenindividuellen (Defekt-)Gegebenheiten, mittels parametrisch veränderbarem Basismodell generiert. Dieser Prozess wurde vorerst für ein nicht-hybrides Implantat entwickelt und validiert und wird als nächstes auf ein hybrides Implantat, konkret auf hybride Wirbelsäulen-Cages angewandt. Angestrebt wird ein integraler, reproduzierbarer Fertigungsprozess, welcher die Kombination von Füll- und Tragstruktur sicherstellt.

\subsection{Diskussion}

Beide Prozesse haben sich für den jeweiligen Anwendungsfall als zielführend erwiesen. Es hat sich gezeigt, dass ein Prozess allein nicht ausreicht, um Hybrid-Implantate zeit- und kosteneffizient zu konstruieren. Je nach Erkrankung und Häufigkeit ihres Auftretens, ist entweder die Verwendung eines parametrischen Basismodells oder die individuelle Modellierung von 
Implantaten sinnvoll. Bei häufig vorkommenden Erkrankungen, die den Austausch eines Knochen- oder Knorpelanteils erfordern, ist die Verwendung eines Basismodells empfehlenswert, wenn der Defekt oder die Dysfunktion die Erneuerung eines globalen und patientenübergreifend gleichen Bereiches erfordert. Als Beispiele sind hier Hüftschaft, Knie oder Wirbel zu nennen. Die zu ersetzenden Bereiche sind patientenunabhängig in Form und Art überwiegend gleich und unterscheiden sich in ihrer Größe und bei kleineren Konturen. Defekte, die hingegen lediglich eine lokale, exakt der Defektgröße entsprechende Implantation erfordern, eignen sich nicht für eine automatisierte, parametrische Lösung. Der automatisierte Prozess basiert auf einem Basismodell, welches sich zwar an den Patientdaten orientiert, allerdings in seiner Dimension festgelegt ist. Defekte im Schädel, bspw. am Schädeldach oder dem Jochbein, sind völlig individuell und treten in der Form selten ein zweites Mal auf. Bei derartigen Defekten werden nur die Bereiche durch neue Implantate ersetzt, die auch erkrankt oder defekt sind.

Grundsätzlich lässt sich nicht für jeden Defekt pauschal festlegen, welcher Prozess der zeitund kosteneffizientere ist. Mit fortschreitender Technik, einem größeren Portfolio an funktionalen Oberflächen und Strukturen sowie der stetig verbesserten und besser auf die patientenindivuellen Anforderungen abgestimmten Implantaten, lassen sich zukünftig womöglich größere Knochen- oder Knorpelanteile mit längerer Einsatzdauer implantieren, wodurch die Verwendung automatisierter Hybridimplantate in weitaus mehr Bereichen sinnvoll sein kann.

\section{Zusammenfassung und Ausblick}

Die additive Fertigung stellt für die Entwicklung und Fertigung individueller Knochenersatzimplantate eine gute Voraussetzung dar. Durch die Verwendung mehrerer Materialien können unterschiedliche Funktionen integriert werden. Ebenso sind auf den Patienten abgestimmte Geometrien umsetzbar. Ein vielversprechender Ansatz ist die Aufteilung der Komponenten in eine lasttragende sowie eine bioaktive Struktur.

Im Rahmen dieser Arbeit konnte ein Prozess entwickelt werden, der als Leitfaden zur Konstruktion hybrider Implantate anwendbar ist. Neben der Konstruktion eines hybriden Schädeldachimplantats wurde dies erfolgreich gefertigt. Sowohl die Auslegung der Tragstruktur als auch die Dimensionierung der Füllstruktur haben sich als geeignet erwiesen.

Des Weiteren wurde ein Verfahren zur Automatiserung von Implantaten entwickelt. Der Vergleich des Anwendungsfalls des jeweiligen Prozesses sowie der zeitliche Aufwand, der für die Erstellung eines parametrischen Basismodells notwendig ist, haben gezeigt, dass eine Automatisierung nicht generell zielführend ist. Bei patientenübergreifend nicht regelmäßig auftretenden Defekten ist das Basismodell aus Zeit- und damit einhergehend Kostengründen nicht zweckmäßig. Ferner wird bei dem Einsatz eines Basismodells prinzipiell der gleiche Knochenoder Knorpelbereich ersetzt. Er unterscheidet sich lediglich in Kontur und Größe. Dennoch bedeutet die Entwicklung eines automatisierten Prozesses für die Erstellung von hybriden Implantaten einen erheblichen Mehrwert durch die höhere Reaktionszeit sowie geringere Kosten.

Hieran anknüpfend gilt es für beide Prozesse zu überprüfen, welche fertigungstechnischen Folgen die Qualtität der Implantate beeinflussen. Dies betrifft insbesondere die Verteilung der Füllstruktur beim Eindruckprozess sowie deren Eindruckstrategie. Weiterhin ist zu untersuchen, inwieweit funktionelle Oberflächen auf der Tragstruktur den stabilen Verbund mit der Füllstruktur weiter erhöhen können, wie diese auszulegen sind und ob eine Dimensionierung dieser beim automatisierten Prozess mitberücksichtigt werden kann. Ferner sollten Untersuchungen zu den Stützstrukturen angstellt werden und ob eine andere Auslegung dieser, den Entferungsprozess nach dem Eindrucken der Füllstruktur positiv beeinflussen kann.

\section{Literaturverzeichnis}

[1] Wintermantel, E. und Ha, S.-W.: Medizintechnik - Life Science Engineering. 4. Aufl. Springer Berlin Heidelberg, 2009.

[2] Loew, M.: AE-Manual der Endoprothetik. Berlin, Heidelberg: Springer Berlin Heidelberg, 2010. 
[3] Tidow, U.: Erfassung, Analyse und Darstellung von Risikofaktoren für das Auftreten von Infektionen nach Implantation einer Kranioplastik zur Defektdeckung nach dekompressiver Kraniektomie. Disseratation, Universität zu Lübeck. Lübeck, 2016.

[4] Waldeyer, Anton und Streicher, Johannes: Waldeyer - Anatomie des Menschen. De Gruyter, 2012.

[5] Schiffner, S.: Entwicklung von Konstruktionsprinzipien für individuelle Hybridimplantate. Diplomarbeit, TU Dresden, 2019.

[6] Scheibner, B.: Konstruktion, Fertigung und Evaluierung hybrider Implantate. Diplomarbeit, TU Dresden, 2019.

[7] BIONIKA, Oral and maxillofacial surgery - BIONIKA. [Online]. Verfügbar unter: https://www.bionika.hu/en/oral-and-maxillofacial-surgery/ (Zugriff am: 23. März 2021).

[8] Evonos, Schädelimplantate Evo-Shape mit hoher Biokompatibilität - evonos. [Online]. Verfügbar unter: https://www.evonos.de/products/evo_shape/ (Zugriff am: 13. Januar 2021).

[9] Silony Medical GmbH: Internetauftritt. Leinfelden-Echterdingen, URL: https://www.silony-medical.com/produkte/ roccia/, April 2021.

[10] Schröder, J.; Herbort, M.; Rustemeyer, P.; Vieth, V.; Wassmann, H.: Mechanical Response of Cervical Vertebral Endplates to Axial Loading. George Thieme Verlag Stuttgart, New York, 2006, DOI: 10.1055/s-2006942279.

[11] Fraunhofer Institut für Lasertechnik (ILT): Design und additive Herstellung lokal lastangepasster Wirbelkörperimplantate. Aachen, Jahresbericht 2017, 2017.

[12] Lim, K.-M.; Park, T.-H.; Lee, S.-J.; Park, S.-J.: Design and Biomechanical Verification of Additive Manufactured Composite Spinal Cage Composed of Porous Titanium Cover and PEEK Body. MDPI, Basel, Switzerland, 2019.

[13] Wild, M.; Schumacher, R.; Mayer, K.; Schkommodau, E.; Thoma, D.; Bredell, M.; Gujer, A. K.; Grätz, K. W.; Weber, F. E.: Bone Regeneration by the Osteoconductivity of Porous Titanium Implants Manufactured by Selective Laser Melting: A Histological and Micro Computed Tomography Study in the Rabbit. Tissue Engineering Part A, Bd. 19, pp. 2645-2654, 122013.

[14] Habijan, T.; Haberland, C.; Meier, H.; Frenzel, J.; Wittsiepe, J.; Wuwer, C.; Greulich, C.; Schildhauer, T. A.; Köller, M.: The biocompatibility of dense and porous Nickel-Titanium produced by selective laser melting. Ma-terials Science and Engineering: C, Bd. 33, pp. 419-426, 12013.

[15] Roth, A.: Periprothetische Osteopenie. Osteologie 2015, George Thieme Verlag, DOI: 10.1055/s-00371622063, 2018.

[16] Schmitt, R.: Werkstoffverhalten in biologischen Systemen. Springer Berlin Heidelberg, 2013.

[17] Lethaus, B.; Safi, Y.; Laak-Poort, M.; Kloss-Brandstätter, A.; Banki, F.; Robbenmenke, C.; Steinseifer, U.; Kess-ler, P.: Cranioplasty with Customized Titanium and PEEK Implants in a Mechanical Stress Model. Journal of Neurotrauma, Bd. 29, pp. 1077-1083, 42012.

[18] Ng, Z. Y.; Nawaz, I.: Computer-Designed PEEK Implants. Journal of Craniofacial Surgery, Bd. 25, 12014.

[19] OssDsign AB: Internetauftritt. Uppsala, Sweden, URL: https://www.ossdsign.com/home, April 2021.

[20] INNOTERE GmbH: Internetauftritt. Radebeul, URL: https://www.innotere.de/, April 2021.

[21] Heinemann, S.; Rössler, S.; Lemm, M.; Ruhnow, M.; Nies, B.: Properties of injectable ready-to-use calcium phosphate cement based on water-immiscible liquid. Acta Biomaterialia 2013, 2013.

[22] Schulz, M. C.; Holtzhausen, S.; Nies, B.; Heinemann, S.; Lauer, G.; Sembdner, P.: Three-dimensional plotted bone calcium-phosphate scaffolds for bone defect augmentation - a new option for regeneration? INTERNATIONAL OSTEOLOGY SYMPOSIUM BARCELONA, 2019.

[23] Kilian, D.; Holtzhausen, S.; Sembdner, P.; Ahlfeld, T.; Akkineni, A. R.; Lode, A.; Stelzer, R.; Gelinsky, M.: 3D Bioprinting of volumetric osteochondral tissue substitutes with an individualized design and an anisotropic inter-nal architecture. 30th Annual Conference of the European Society for Biomaterials together with the 26th Annual Conference of the German Society for Biomaterials (DGBM), 2019.

[24] Holtzhausen, S.; Heinemann, S.; Lemm; M.; Stelzer, R.: Printing of contour-adapted bone scaffolds based on calcium phosphate cements. CARS 2019, 2019.

[25] Reitmaier, S.; Kovtun, A.; Schuelke, J.; Kanter, B.; Lemm, M.; Hoess, A.; Heinemann, S.; Nies, B.; Ignatius, A.: Strontium(II) and Mechanical Loading Additively Augment Bone Formation in Calcium Phosphate Scaffolds. Journal of Orthopaedic Research Society, 2017.

[26] Sembdner, P. et al.: Design of a parametric knee implant model based on Active Shape Model output data for individualized knee implants. In: CARS 2019, 2019.

[27] Sembdner, P.: Rechnergestützte Planung und Rekonstruktion für individuelle Langzeit-Knochenimplantate am Beispiel des Unterkiefers. Dissertation, TU Dresden, 2017.

[28] Mika, L. et al.: Process Optimization for the Manufacturing of Individualized Ankle Foot Orthoses via Digitalization and AM. In: ASME, MSEC 2021. University of Cincinnati, 2021.

[29] Mika, L. et. al.: Konstruktion und Auslegung von Biegegelenken für die Herstellung individueller Unterschenkelorthesen mittels additiver Fertigung. In: Konstruktion für die Additive Fertigung 2020. Hannover, 2020. 